\title{
Optimization of the Coupling Coefficient of the Inductive Link for Wireless Power Transfer to Biomedical Implants
}

\author{
Jiarui Bao, Shuyan Hu, Zibin Xie, Guangxi Hu (D), Ye Lu (iD, and Lirong Zheng \\ School of Information Science and Technology, Fudan University, Shanghai 200438, China \\ Correspondence should be addressed to Guangxi Hu; gxhu@fudan.edu.cn and Ye Lu; lu_ye@fudan.edu.cn
}

Received 31 October 2021; Accepted 9 December 2021; Published 6 January 2022

Academic Editor: Paolo Baccarelli

Copyright (C) 2022 Jiarui Bao et al. This is an open access article distributed under the Creative Commons Attribution License, which permits unrestricted use, distribution, and reproduction in any medium, provided the original work is properly cited.

\begin{abstract}
This work focuses on the optimization of coupling coefficient $(k)$ of the inductive link for the wireless power transfer (WPT) system to be used in implantable medical devices (IMDs) of centimeter size. The analytic expression of $k$ is presented. Simulations are conducted by using the high-frequency structure simulator (HFSS). Analytic results are verified with simulations. The receiving $(\mathrm{Rx})$ coil is implanted in the body and set as a circular coil with a radius of 5 millimeters for reducing the risk of tissue inflammation. The inductive link under misalignment scenarios is optimized to improve $k$. When the distance between the transmitting ( $\mathrm{Tx}$ ) and $\mathrm{Rx}$ coils is fixed at $20 \mathrm{~mm}$, it is found that, to maximize $k$, the Tx coil in a planar spiral configuration with an average radius of $20 \mathrm{~mm}$ is preferred, and the Rx coil in a solenoid configuration with a wire pitch of $0.7 \mathrm{~mm}$ is recommended. Based on these optimization results, an inductive link WPT system is proposed; the coupling coefficient $k$, the power transfer efficiency (PTE), and the maximum power delivered to the load (MPDL) of the system are obtained with both simulation and experiment. Different media of air, muscle, and bone separating the Tx and Rx coils are tested. For the muscle (bone) medium, PTE is $44.14 \%(43.07 \%)$ and MPDL is $145.38 \mathrm{~mW}(128.13 \mathrm{~mW})$, respectively.
\end{abstract}

\section{Introduction}

Implantable medical devices (IMDs) have been widely used in the clinical society, such as cardiac pacemakers, cochlear implants, left vagus nerve stimulators, spinal cord stimulators, retinal implants, and deep brain stimulators [1-9]. At present, most IMDs are powered by batteries for the convenience and comfort of patients. The IMD has to be replaced with a new one by surgery when the battery is out of energy, which may be quite painful for the patients. Wireless power transfer (WPT) has emerged as a promising solution for these drawbacks, enable minimally invasive long-term implantation of those devices, and eliminate the need for another surgery for IMD replacement.

There are several techniques to realize WPT, such as ultrasound, optical, microwave radiation, electric field coupling, and inductive coupling [10-18]. Among these methods, inductive coupling is the most preferred for IMDs, where power is transmitted from one coil and received by the other (usually integrated with the IMD inside a human body) through electromagnetic induction.
To enhance the power transfer efficiency (PTE), the inductive coupling coefficient $(k)$ between the transmitting (Tx) and receiving ( $\mathrm{Rx}$ ) coils needs to be maximized. As a key parameter of the WPT system, $k$ can be obtained with the relationship $k=M / \sqrt{L_{1} L_{2}}$, where $L_{1}$ and $L_{2}$ are the self-inductances of the Tx and Rx coils, respectively, and $M$ is the mutual inductance, which typically depends on the geometries of the two coils, their distance, and the material separating them.

Existing works focused on the mutual inductance for the coils in different geometries, such as a solenoid, thin disk, filamentary circular, and spiral rectangular coils [19-30]; as well as in different relative positions, such as coaxial, noncoaxial, and inclined axial [31-33]. In addition, some works focused on calculating the self-inductance of the coils in different geometries, such as square, hexagonal, octagonal, and circular planar [34,35]. Analytical expressions for selfinductance were obtained in $[36,37]$. The self-inductance and mutual inductance of the printed square-shaped spiral coils were explored, which were used to design an inductive WPT system [38]. 
To avoid or eliminate the side effects of large-sized IMDs, such as inflammation, cell death, and users' pain, the size of the IMD has been continuously scaling down $[17,18]$. With the rapid development in integrated circuit (IC) technologies, IMDs for cardiac pacemakers or nerve stimulators can likely be miniaturized to a centimeter (cm) scale [42]. Therefore, the dimension of the Rx coil is limited, and PTE and the maximum power delivered to the load (MPDL) are reduced. New WPT systems have to be designed for $\mathrm{cm}$-sized IMDs to enhance the coupling coefficient, which plays a critical role in improving the PTE and MPDL.

An LCC-C compensated WPT system for cardiac pacemakers was designed in [39], and the size of the designed system to be embedded was quite large, $31.4 \mathrm{~mm} \times 47.4 \mathrm{~mm} \times 8.1 \mathrm{~mm}$, which was against the spirit of minimally invasive applications. The distance between the Tx and Rx coils was only $8 \mathrm{~mm}$, which may be too short for real-world usage. A sandwiched WPT system was designed for recharging the battery of a micromedical robot [40]. The system is quite complicated and is inconvenient for the patient to use.

For midrange $(\sim 2 \mathrm{~m})$ inductive link power transmission, it is necessary to transmit power at resonance to reduce the dissipated energy in the objects between the Tx and $\mathrm{Rx}$ coils and improve the transmission efficiency [41]. For the short-range $(\sim 2 \mathrm{~cm})$ power transmission, power losses in the Tx and Rx coils become significant. Resonance is generally employed to reduce power and tissue losses and improve the PTE $[42,43]$.

In this paper, we aim to optimize the coupling coefficient of an inductive link, where the operating frequency, the configurations, and sizes of the Tx and Rx coils are selected to maximize $k$. After that, we propose a WPT system with the optimized link for $\mathrm{cm}$-sized IMDs. We consider two types of candidate Tx coils, namely, single-turn circular coil and planar spiral circular coil. We examine the mutual inductances of Tx-Rx, and in turn, the coupling coefficients, with the Tx coil in various sizes through extensive simulations, under different Tx-Rx coil distances, operating frequency, wire pitch of the $\mathrm{Rx}$ coil, and turn number of the Tx coil. According to the optimized results, for the specific applications such as a cardiac pacemaker, the planar spiral coil is selected as the Tx coil for the WPT system, along with other optimized parameters for the Tx and Rx coil configurations. We proceed to investigating the PTE and MPDL of the planar spiral Tx coil when the Tx and Rx coils are separated with different media, including air, muscle, and bone. At last, we carry out experiments to corroborate the simulation results and validate the efficiency of the proposed WPT system in real-world applications. Such WPT systems can largely extend the lifespan of $\mathrm{cm}$-sized IMDs and greatly benefit the patients.
We focus on the power transmission for IMDs, and data communication is out of the scope of this work and will be an interesting research direction for future works.

The novelties of the present work are summarized as follows:

(1) The optimizations of the coupling coefficient of an inductive link WPT system for $\mathrm{cm}$-sized IMDs are presented, with the misalignment scenarios of the inductive link coils included.

(2) Based on the optimizations of $k$, an inductive link WPT system with the $\mathrm{Rx}$ coil in $\mathrm{cm}$ size is proposed, which can be applied to recharge the button cell in a cm-sized IMD.

(3) Simulation results of $k$, PTE, and MPDL of the proposed inductive link WPT system are verified against experiments for different media separating the $\mathrm{Tx}$ and $\mathrm{Rx}$ coils, for example, air, muscle, and bone.

(4) The deembedding calibration method is adopted in experiments. When the operation frequency is $25 \mathrm{MHz}$, for the muscle (bone) medium, a PTE of $44.14 \%(43.07 \%)$ and an MPDL of $145.38 \mathrm{~mW}$ $(128.13 \mathrm{~mW})$ are demonstrated with measurements.

The rest of the paper is arranged as follows: in Section 2, the theoretical expressions of $k$, PTE, and MPDL for an inductive link WPT system are presented. In Section 3, extensive simulations are provided. Different inductive link models are optimized by simulations, and an inductive link WPT system is proposed. In Section 4, experimental systems of the WPT links are set up, and the measured results are obtained by the using deembedding calibration method. Discussions are provided in Section 5, and concluding remarks are presented in Section 6.

\section{Analytical Model}

2.1. Single-Turn Circular Tx Coil. For the Tx coil, a single-turn coil can be used to reduce the specific absorption rate (SAR) and increase MPDL [17]. As a multiturn coil can be equaled to a set of single-turn coils, we only deal with a single-turn Tx coil in this work. Figure 1(a) shows the model of a coaxial WPT system with a single-turn circular Tx coil. The inductive link consists of a Tx coil and an Rx coil. For an implantable WPT system, the $\mathrm{Rx}$ coil should be small sized as aforementioned, so $R_{2}$ is limited to $5 \mathrm{~mm}$ in this paper. The Tx coil is placed outside the body to be excited to generate magnetic fluxes. A portion of the fluxes is picked up by the implanted $\mathrm{Rx}$ coil. In this way, power can be transmitted wirelessly, overcoming the obstacles of the human body. The tangential component of the vector magnetic potential at the location of the $\mathrm{Rx}$ coil can be expressed as [31, 44]

$$
A_{\phi}\left(R_{1}, R_{2}, h\right)=\frac{\mu_{0} I_{1}}{2 \pi} \frac{\sqrt{\left(R_{1}+R_{2}\right)^{2}+h^{2}}}{R_{2}}\left[\left(1-\frac{1}{2} w^{2}\right) K(w)-G(w)\right]
$$




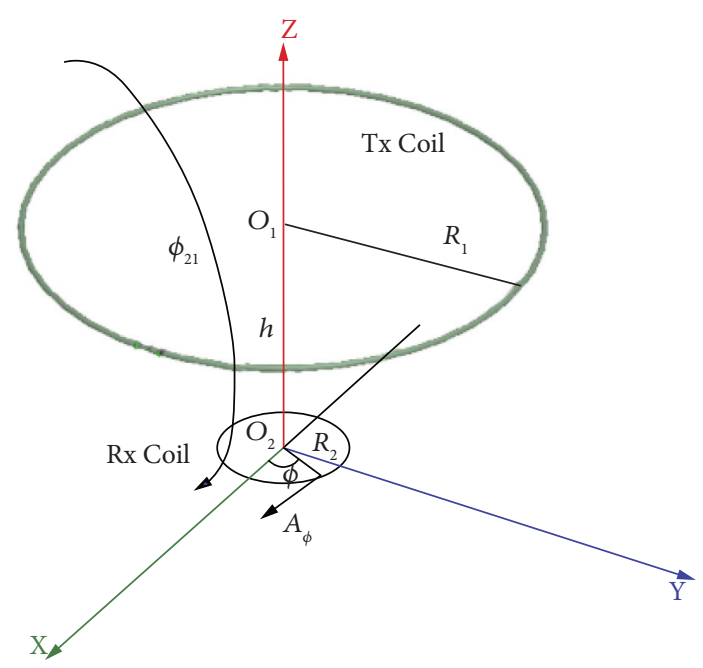

(a)

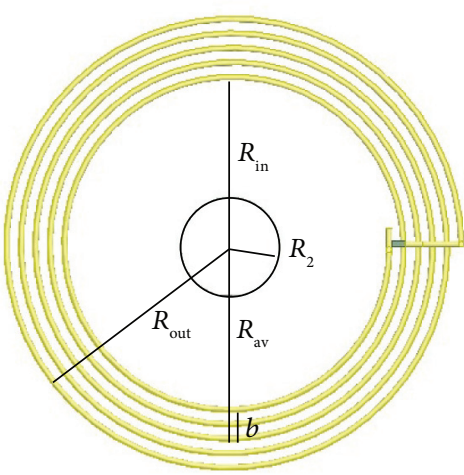

(b)

Figure 1: (a) The inductive link system model with a single-turn Tx coil. (b) The planar spiral circular Tx coil in HFSS simulator.

where $h$ is the spacing between the two coils, $R_{1}$ and $R_{2}$ are the radius of Tx and $\mathrm{Rx}$ coils, respectively, and $I_{1}$ is the excitation current in the Tx coil.

$$
K(w)=\int_{0}^{\pi / 2} \frac{1}{\sqrt{1-w^{2} \sin ^{2} \psi}} \mathrm{d} \psi
$$

is the second kind of complete elliptic integral,

$$
G(w)=\int_{0}^{\pi / 2} \sqrt{1-w^{2} \sin ^{2} \psi} d \psi
$$

is the first kind of complete elliptic integral, and

$$
w \equiv 2 \sqrt{\frac{R_{1} R_{2}}{\left[\left(R_{1}+R_{2}\right)^{2}+h^{2}\right]}},
$$

is the modulus of the elliptic integral. $\mu_{0}$ is the permeability in the vacuum.

The magnetic flux excited on the Rx coil by the Tx coil can be obtained as [31]

$$
\phi_{21}=\iint_{S_{2}} \vec{B} \cdot \mathrm{d} \vec{S}_{2}=\iint_{S_{2}}(\nabla \times \vec{A}) \cdot \mathrm{d} \vec{S}_{2}=\oint_{l_{2}} \vec{A} \cdot \mathrm{d} \vec{l}_{2}=2 \pi R_{2} A_{\phi}
$$

Then, the mutual inductance, $M$, can be written as $[45,46]$

$$
M=\frac{\phi_{21}}{I_{1}}=2 \pi R_{2} \frac{A}{I_{1}}=\frac{2 \mu_{0} \sqrt{R_{1} R_{2}}}{w}\left[\left(1-\frac{w^{2}}{2}\right) K(w)-G(w)\right] .
$$

The self-inductance of the filamentary circular coil, $L_{f c c}$, is given by [47]

$$
L_{f c c}=\mu_{0} R\left[\ln \left(\frac{8 R}{r}\right)-2+\frac{p}{4}\right]+\mu_{0} O\left(\frac{r^{2}}{R}\right),
$$

where $R$ is the radius of the coil, for the Tx and Rx coils, it is $R_{1}$ and $R_{2}$, respectively. $r$ is that of the wire, and the constant $p$ depends on the distribution of the current at the cross-section of the wire: $p=1$ when the current is uniformly distributed, and $p=0$ when there is an obvious electric current skin effect. Under high operating frequencies $(>10 \mathrm{MHz})$, the skin effect will be large and $p=0 . O(\ldots)$ stands for a small term, when $R \gg r$, the term $\mu_{0} O\left(r^{2} / R\right)$ is negligible, then we have

$$
L_{f c c}=\mu_{0} R\left[\ln \left(\frac{8 R}{r}\right)-2\right]
$$

With equations (6) and (8), we can obtain the coupling coefficient of the single-turn circular Tx and Rx coils case as follows:

$$
k_{s t}=\frac{2 \sqrt{R_{1} R_{2}}}{D w}\left[\left(1-\frac{w^{2}}{2}\right) K(w)-G(w)\right],
$$

where

$$
D \equiv \sqrt{R_{1} R_{2}\left[\ln \left(\frac{8 R_{1}}{r_{1}}\right)-2\right]\left[\ln \left(\frac{8 R_{2}}{r_{2}}\right)-2\right]} .
$$

2.2. Planar Spiral Circular Tx Coil. Usually, a planar spiral Tx coil can be used to enhance the coupling coefficient [38]; therefore, we deal with this type of configuration for the Tx coil. Figure 1(b) shows the WPT system established in HFSS, and the Tx coil is configured as a planar spiral. In the 
model, $R_{\mathrm{in}}$ and $R_{\text {out }}$ are the inner and outer radii of the Tx coil, respectively. The average radius $R_{a v}$ equals to $0.5\left(R_{\text {out }}+R_{\text {in }}\right), b$ equals to $0.5\left(R_{\text {out }}-R_{\text {in }}\right)$, and $n_{1}$ is the turn number of the coil. Then, the mutual inductance of the Tx coil with a single-turn circular Rx coil, $M_{p}$, can be obtained as $[31,33]$

$$
M_{p}=\frac{n_{1}}{(2 n+1)} \sum_{l=-n}^{n} M(l)
$$

where $n=1,2,3, \ldots$ is a positive integer, and a larger value of $n$ leads to a more precise result of $M_{p}$.

$$
M(l)=\frac{\mu_{0} \sqrt{R_{1}^{\prime} R_{2}}}{w^{\prime}}\left[\left(2-w^{\prime 2}\right) K\left(w^{\prime}\right)-2 G\left(w^{\prime}\right)\right],
$$

where

$$
\begin{aligned}
& R_{1}^{\prime} \equiv R_{a v}+\frac{2 b}{(2 n+1)} l, \\
& w^{\prime}=2 \sqrt{\frac{R_{1}^{\prime} R_{2}}{\left(\left(R_{1}^{\prime 2}+R_{2}\right)^{2}+h^{2}\right)}} .
\end{aligned}
$$

The self-inductance of a planar spiral coil, $L_{p s c}$, can be written as [36]

$$
L_{p s c}=\mu_{0} R_{a v} n_{1}^{2}\left[\ln (2.46) \rho+0.2 \rho^{2}\right],
$$

where $\rho=b / R_{a v}$. The $k$ of the planar spiral circular Tx coil with the aforementioned $\mathrm{Rx}$ coil $k_{p s}$ can be obtained as

$$
k_{p s}=\frac{M_{p}}{\sqrt{L_{1} L_{2}}}
$$

where $M_{p}$ can be obtained with equation (11), and $L_{1}$ and $L_{2}$ can be obtained with equations (8) and (15), respectively.

2.3. PTE and MPDL. Figure 2 shows the illustration of the circuit model for an inductive link WPT system. $R_{T x}, R_{R x}$, and $R_{L}$ are the resistances of the Tx coil, the Rx coil, and the load, respectively. $C_{1}$ and $C_{2}$ are the capacitors. It should be noted that the values of $R_{T x}$ and $R_{R x}$ are frequency-dependent, owing to the electric current skin effect under high frequency. The PTE, denoted with $\eta$, is defined as the ratio of the power received by the load, $P_{L}$, over the power input to the Tx coil, $P_{i n}$, and at resonance $\eta$ can be expressed as $[18,48]$

$$
\eta=\frac{P_{L}}{P_{\text {in }}}=\frac{k^{2} Q_{1} Q_{2}}{\left(1+Q_{L} / Q_{2}\right)\left(1+Q_{2} / Q_{L}+k^{2} Q_{1} Q_{2}\right)},
$$

where $Q_{1}=\omega L_{1} / R_{T x}, Q_{2}=\omega L_{2} / R_{R x}$, and $Q_{L}=R_{L} /\left(\omega L_{2}\right)$ are the quality factors of the $\mathrm{Tx}$, the $\mathrm{Rx}$, and the load, respectively. $\omega$ is the angular frequency.

The PDL can be obtained as follows [18]:

$$
P_{L}=P_{\text {in }} \eta=\frac{1}{2} I_{1}^{2}\left(R_{T x}+R_{r e f}\right) \eta,
$$

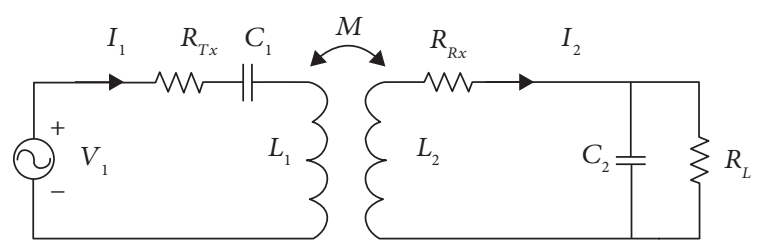

FIgURE 2: The illustration of the circuit model for an inductive link power transfer system.

where $R_{r e f}$ is the reflected impedance to account for the effect of the Rx coil on the Tx coil at resonance and is expressed as follows:

$$
R_{r e f}=\frac{k^{2} \omega L_{1} Q_{2} Q_{L}}{Q_{2}+Q_{L}}
$$

It should be noted that PDL increases with the square of $I_{1}$, but on the other hand, the SAR increases also with the square of $I_{1}$. The local SAR limit for a human body is $2 \mathrm{~W} / \mathrm{kg}$ [49]. Typically, energy absorption occurs on the tissue surface, and the peak value of SAR, denoted with $\mathrm{SAR}_{p}$, is assumed to be the maximum radiation value when the $\mathrm{Tx}$ coil is driven by a constant current, $I_{1}=1 \mathrm{~A}$ in this work. Therefore, the maximum current in the Tx coil within the SAR limit can be expressed as [50]

$$
I_{1, \max }=1(\mathrm{~A}) \sqrt{\frac{2.0(\mathrm{~W} / \mathrm{kg})}{S A R_{p}}} .
$$

Then, the MPDL is

$$
P_{L, \text { max }}=\frac{1}{2} I_{1, \max }^{2}\left(R_{T x}+R_{r e f}\right) \eta .
$$

With equations (17) and (21), PTE and MPDL can be obtained.

\section{Simulation Results}

3.1. Single-Turn Circular Tx Coil. It is said that, for the cmsized IMDs, the operating frequency of a few tens of $\mathrm{MHz}$ is good for both PTE and SAR-constrained PDL. If the frequency is raised, the PTE would be enhanced; but on the other hand, the SAR-constrained PDL would be depressed, and vice versa [18]. Therefore, the operating frequency range in this work is from $10 \mathrm{MHz}$ to $60 \mathrm{MHz}$.

Figure 3(a) shows $M$ versus $R_{1}$ of the Tx coil with different values of $h$, and $R_{2}$ is assumed to be $5 \mathrm{~mm}$. It is noted in the figure that when the value of $h$ is smaller, which means $\mathrm{Tx}$ is closer to $\mathrm{Rx}, \mathrm{Rx}$ will receive more magnetic fluxes emitted from the Tx coil, leading to a larger value of $M$. Figure 3(b) illustrates $k$ versus $R_{1}$ at the operation frequency $f_{p}=30 \mathrm{MHz}$. It is observed that, as the value of $h$ increases, the maximized $k$ can be obtained by selecting a proper value of $R_{1}$. Moreover, when $h$ is large ( $>12 \mathrm{~mm}$ ), the effect of the variation of $R_{1}$ on $k$ will be small. If the distance between the Tx and Rx coils is set as $20 \mathrm{~mm}$, the maximum $k$ of $0.013 \mathrm{can}$ be obtained when $R_{1}=20 \mathrm{~mm}$.

The effect of the Tx coil deformed from a circular circle to two ellipses is investigated. For an ellipse, the aspect ratio is 


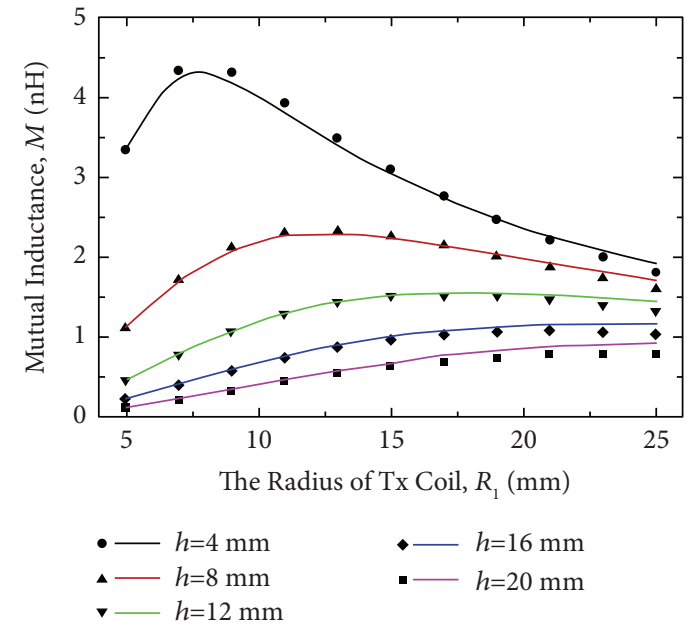

(a)

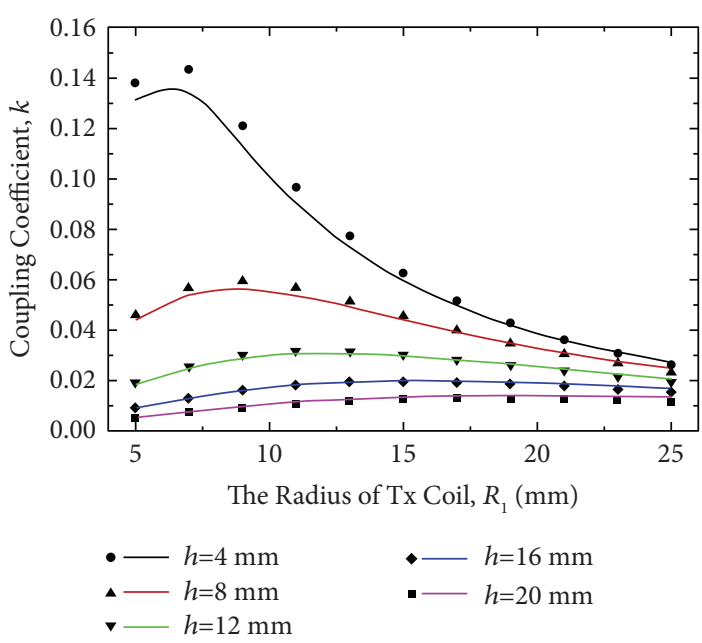

(b)

Figure 3: Simulation and analytical results for (a) $M$ of the WPT system and (b) $k$ of the WPT system. The radius of the Tx coil, $R_{1}$ varies from 5 to $25 \mathrm{~mm}$, while the spacing between the two coils, $h$ is swept from 4 to $20 \mathrm{~mm}$. Symbols and lines represent the simulation and analytical results, respectively. $R_{2}=5 \mathrm{~mm}, r_{1}=0.287 \mathrm{~mm}, r_{2}=0.04 \mathrm{~mm}$, and $f_{p}=30 \mathrm{MHz}$.

defined as the ratio of the major axis length over minor axis length, and the aspect ratios of the two ellipses are set to be 1.5 and 2, respectively. The radius of the circular coil is $20 \mathrm{~mm}$, and the circumference of every coil is $126 \mathrm{~mm}$. The distance between the Tx and Rx coils is $20 \mathrm{~mm}$. Figure 4 shows the simulation results of value $k$ between one of the three shapes of a Tx coil and an Rx coil, respectively. It is observed that, $k$ will decrease with the increase of the aspect ratio. For instance, when $f_{p}=25 \mathrm{MHz}, k$ is 0.0121 for the circular circle of the Tx coil, $k$ drops to 0.0120 and 0.0113 for the elliptic coils of the aspect ratio of 1.5 and 2, respectively. Therefore, a deformed circular Tx coil leads to a smaller value of $k$.

Figure 5 shows the simulation results of $k$ with multiturn $\mathrm{Rx}$ coils. The turn number of the $\mathrm{Rx}$ coil, $n_{2}$, is swept from 2 to 6 , and the wire pitch of the coil, $s$, is swept from $0.1 \mathrm{~mm}$ to $1.1 \mathrm{~mm}$. It is revealed that the coupling coefficient $k$ increases with $n_{2}$. On the other hand, as $s$ increases, $k$ first increases and then decreases, and peaks around $s=0.7 \mathrm{~mm}$. The Tx coil is a single-turn circular coil with $R_{1}=20 \mathrm{~mm}$, and $r_{1}=0.287 \mathrm{~mm}$.

3.2. Planar Spiral Circular Tx Coil. For the planar spiral circular Tx coil case, we can obtain the mutual inductance between the Tx and Rx coils with equation (11), and the self-inductance of the Tx coil with equation(15). After that, with the use of equation(16), we can obtain $k$.

Figure 6 depicts the $k$ value versus the turn number of the Tx coil, $n_{1}$, with different $b$ at $f_{p}=30 \mathrm{MHz}$. Simulations show that, $k$ increases slightly with $n_{1}$, and/or with $b$. The reason is that, when $R_{a v}$ is fixed, as $b$ increases, $R_{\text {out }}$ will be increased while $R_{\text {in }}$ will be reduced; then a few more magnetic fluxes emitted from the Tx coil will be collected by the $\mathrm{Rx}$ coil, as such, $k$ increases slightly with $b$. Therefore, with a fixed value of $R_{a v}$, a large $b$ of the Tx coil is preferred.

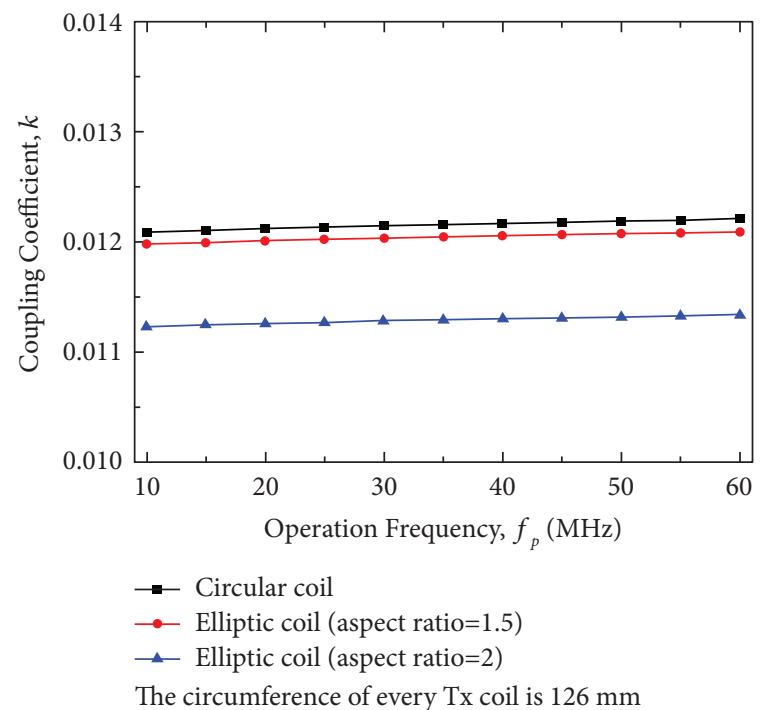

FIGURE 4: Simulation results of $k$ between one of the three shapes of a single-turn Tx coil and an $\mathrm{Rx}$ coil. For the Rx coil, $R_{2}=5 \mathrm{~mm}$, $r_{1}=0.287 \mathrm{~mm}, r_{2}=0.04 \mathrm{~mm}$, and $n_{2}=1$. The distance between the $\mathrm{Tx}$ and $\mathrm{Rx}$ is $20 \mathrm{~mm}$.

\subsection{Optimizations of Tx and Rx Coils for the Misalignment} Scenarios. The above researches are based on the premise that the $\mathrm{Tx}$ and $\mathrm{Rx}$ coils are perfectly aligned, and they are coaxial. However, in practice, misalignment may happen in two ways as illustrated in Figure 7: (1) horizontal distance deviation, where the two coils are not coaxial, and the distance between the two axes of the coils is $d_{0}$, and (2) vertical angle deviation $(\theta)$ of the Rx coil, where the two coils are not parallel to each other. When $d_{0}=0$ and $\theta=0$, the two coils are perfectly aligned. We use HFSS to simulate the inductive link of the two misalignment cases. The parameters optimized in Section 3.2 are adopted, i.e., $R_{a v}=20 \mathrm{~mm}, n_{1}=7, b=6 \mathrm{~mm}, r_{1}=0.287 \mathrm{~mm}, R_{2}=5 \mathrm{~mm}$, $r_{2}=0.04 \mathrm{~mm}, h=20 \mathrm{~mm}$, and $f_{p}=30 \mathrm{MHz}$. 


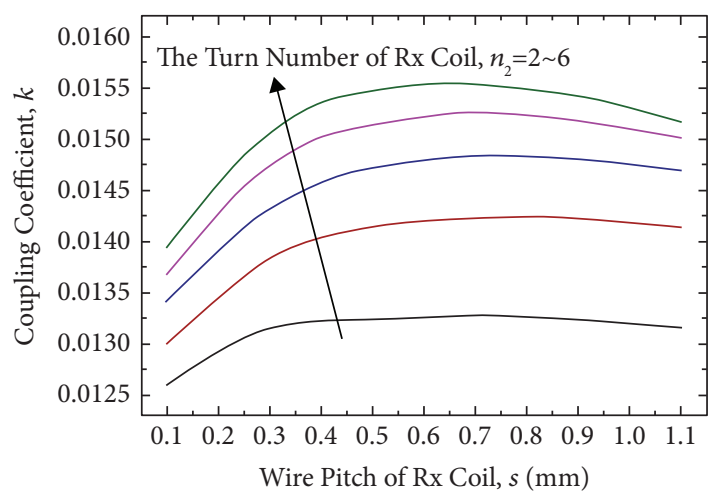

FIGURE 5: Simulation results for $k$ of WPT with a solenoid Rx coil. The turn number of $\mathrm{Rx}$ coil $n_{2}$ is swept from 2 to 6 , and the wire pitch of Rx coil $s$ is swept from $0.1 \mathrm{~mm}$ to $1.1 \mathrm{~mm}$. A single-turn circular Tx coil is used, $R_{1}=20 \mathrm{~mm}$ and $r_{1}=0.287 \mathrm{~mm}$. For the $\mathrm{Rx}$ coil, $R_{2}=5 \mathrm{~mm}$, and $r_{2}=0.04 \mathrm{~mm} . f_{p}=30 \mathrm{MHz}$ and $h=20 \mathrm{~mm}$.

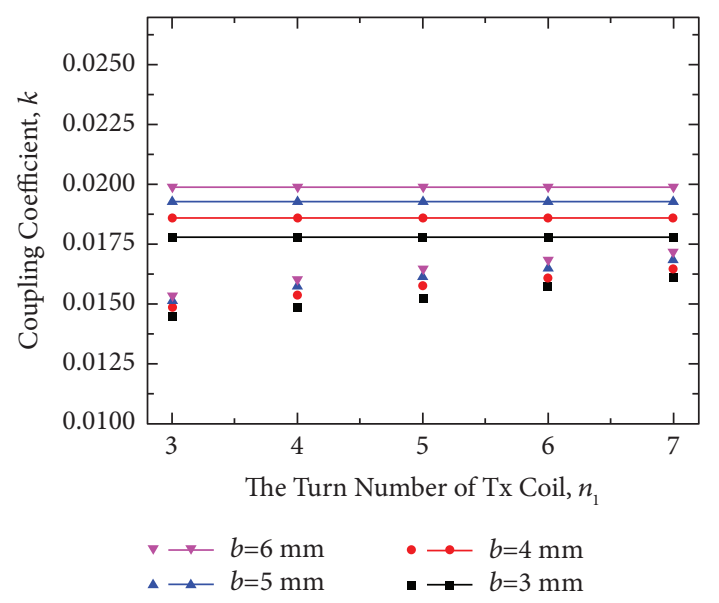

FIGURE 6: Simulated and analytical results of $k$ of a WPT system by sweeping $n_{1}$ and $b$. Symbols are for simulated, while lines with symbols are for analytical, respectively. $f_{p}=30 \mathrm{MHz}, h=20 \mathrm{~mm}$, $R_{a v}=20 \mathrm{~mm}, R_{2}=5 \mathrm{~mm}, r_{1}=0.287 \mathrm{~mm}$, and $r_{2}=0.04 \mathrm{~mm}$.

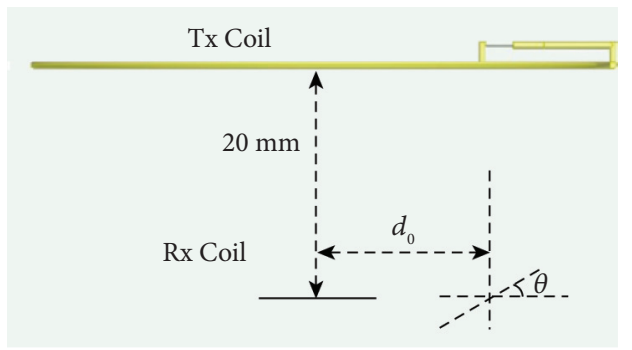

Figure 7: The WPT optimization model in HFSS when the implant has a horizontal offset $\left(d_{0}\right)$ and a vertical angle deviation $(\theta)$.

Figure 8 shows the simulated $k$ of the inductive links when the horizontal deviation distance $\left(d_{0}\right)$ is swept from $0 \mathrm{~mm}$ to $20 \mathrm{~mm}$, and the vertical angle deviation $(\theta)$ of the $\mathrm{Rx}$ coil is swept from $0^{\circ}$ to $90^{\circ}$. It can be seen that the value of $k$ will be greatly reduced by increasing either $d_{0}$ or $\theta$. However, when there is a horizontal distance deviation, $k$ can be improved by introducing a proper vertical angle deviation

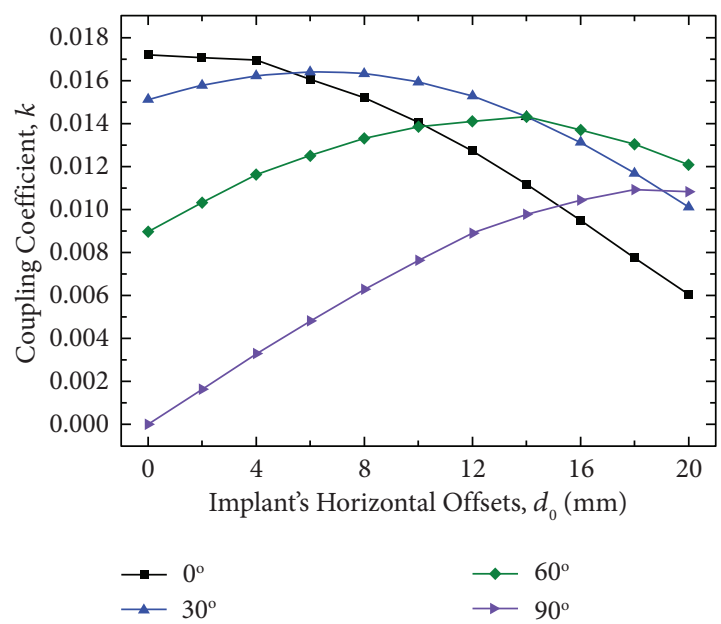

Figure 8: The simulated values of $k$ for the inductive links at $30 \mathrm{MHz}$ versus $\mathrm{Rx}$ coil's horizontal offsets $\left(d_{0}\right)$ and vertical angle deviation $(\theta)$.

intentionally. The reason is that, when the vertical angle deviation is introduced, the two coils are adjusted to be coaxial, and the Rx coil will pick up more magnetic fluxes than before. Similarly, if the Rx coil has a vertical angle deviation, $k$ can be improved by introducing a proper horizontal distance intentionally.

Table 1 shows the simulated values of PTE and MPDL versus vertical angle deviation $(\theta)$, when there is no horizontal distance deviation, $d_{0}=0 \mathrm{~mm}$. It can be seen that the values of PTE and MPDL will decrease with $\theta$. When $\theta=60^{\circ}$, PTE will decrease from $12.29 \%$ to $6.49 \%$, and MPDL will drop from $27.72 \mathrm{~mW}$ to $4.33 \mathrm{~mW}$.

It is noted that observed from Figure Rong et al. [51] have investigated the optimization design of resonance coils for drone wireless charging system, where the horizontal misalignment between the Tx and Rx coils may happen. For the first time, the authors proposed an unequally spaced $\mathrm{Tx}$ coil and a varying radius size per turn $\mathrm{Rx}$ coil to well address the misalignment problem. They obtained a maximum PTE improvement of $56.23 \%$ over traditional coils. In our work, the application is for IMDs, the horizontal and angular misalignments between the Tx and Rx coils may also happen, and the problem can be solved by moving the Tx coil outside the human body.

3.4. A Proposed Inductive Link for the WPT System. Based on the simulation results, an inductive link for the WPT system is proposed, which may be applied to recharging the battery of a cm-sized IMD. With the concern of coupling coefficient, the Tx coil in a planar spiral circular configuration is used. To increase the MPDL, the Rx coil with 5 turns in a solenoid configuration is adopted, and from the results of Figure 5, the wire pitch is set to be $0.7 \mathrm{~mm}$. Typically, the optimal load resistance of an IMD for cardiac implantable electric devices is $1 \mathrm{k} \Omega$ [52], and this load resistance value is assumed in this work. The geometric parameters are listed in Table 2.

Figure 9 demonstrates the maximum current allowed in the Tx coil (solid line with circles) and the peak value of SAR 
TABle 1: Parameters of misaligned coils $\left(f_{p}=30 \mathrm{MHz}\right.$ and $\left.d_{0}=0 \mathrm{~mm}\right)$.

\begin{tabular}{lcccc}
\hline Vertical angle deviation $(\theta)$ & $0^{\circ}$ & $30^{\circ}$ & $60^{\circ}$ & $90^{\circ}$ \\
\hline PTE $(\%)$ & 12.29 & 10.77 & 6.49 & $8.63 \times 10^{-8}$ \\
MPDL (mW) & 27.72 & 19.10 & 4.33 & $6.39 \times 10^{-9}$ \\
\hline
\end{tabular}

TABLE 2: Geometric parameters of a proposed inductive link.

\begin{tabular}{lcc}
\hline Parameters & & Values \\
\hline \multirow{4}{*}{ Tx coil } & Outer radius $\left(R_{\text {out }}\right)$ & $26 \mathrm{~mm}$ \\
& Inner radius $\left(R_{\text {in }}\right)$ & $14 \mathrm{~mm}$ \\
& Wire diameter $\left(r_{1}\right)$ & $0.287 \mathrm{~mm}(\mathrm{AWG} 23)$ \\
& Parameter $b$ & $6 \mathrm{~mm}$ \\
& Number of turns $\left(n_{1}\right)$ & 7 \\
Rx coil & Radius $\left(R_{2}\right)$ & $5 \mathrm{~mm}$ \\
& Wire radius $\left(r_{2}\right)$ & $0.04 \mathrm{~mm}(\mathrm{AWG} 40)$ \\
Tx-to-Rx distance $(h)$ & $0.7 \mathrm{~mm}$ \\
Tx-to-tissue distance & $20 \mathrm{~mm}$ \\
Load resistance $\left(R_{L}\right)$ & $2 \mathrm{~mm}$ \\
\hline \multicolumn{2}{c}{ Wire pitch $(s)$} & $1 \mathrm{k} \Omega$
\end{tabular}

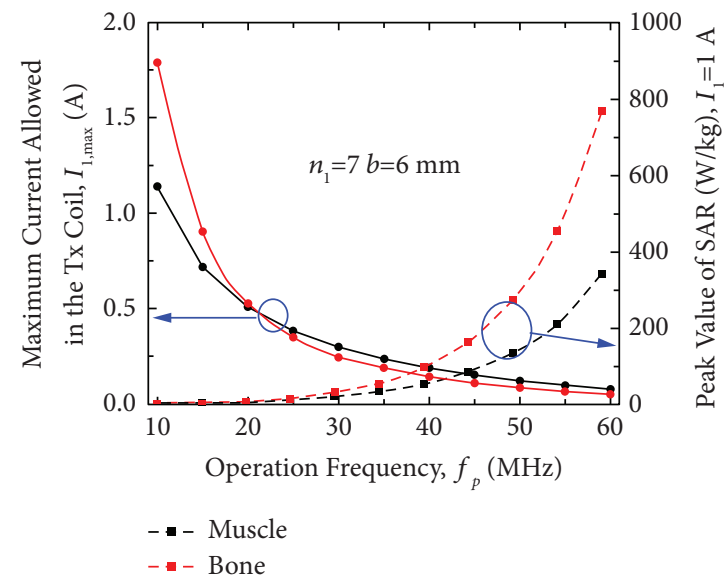

FIgUre 9: At Peak value of SAR at the surface of the tissue, the excitation current in the Tx coil is $1 \mathrm{~A}$ (left). Maximum current allowed in the Tx coil at the peak value of SAR is set to be $2 \mathrm{~W} / \mathrm{kg}$ (right).

at the surface of the tissue (dashed line with squares), which are presented with the left and the right vertical axes, respectively. For the peak value of SAR, the excitation current in the Tx coil is set to be $1 \mathrm{~A}$, while for the maximum current allowed in the Tx coil, the peak value of SAR is assumed to be $2 \mathrm{~W} / \mathrm{kg}$. As expected, the peak value of SAR increases with the frequency, while the maximum current allowed in the Tx coil decreases with frequency.

Figure 10 presents the simulation results of $k$, PTE, and MPDL of the proposed inductive link WPT system, with different media of air, muscle, and bone separating the two coils. The frequency-dependent dielectric properties of bone and muscle are adopted in simulations [53]. Typically, for the frequency of $25 \mathrm{MHz}$, the relative permittivity, the conductivity, and dielectric loss tangent of muscle and bone are listed in Table 3.
Figure 10(a) shows that, the values of $k$ are almost the same with all three different media at low frequencies $(<30 \mathrm{MHz})$. In the frequency range of $30 \mathrm{MHz}<f_{p}<60 \mathrm{MHz}$, there are some differences of values of $k$, the largest is about $3 \%$ which occurs between the air and the muscle for $f_{p}=60 \mathrm{MHz}$. The reason is that, $k$ is associated with the permeability of the medium, which are almost the same for the three media at low frequencies. As frequency increases, so does permeability. The fastest increase of permeability is the muscle, followed by the bone. Therefore, in the frequency range of $30 \mathrm{MHz}<f_{p}<60 \mathrm{MHz}$, there are some differences of values of $k$.

It is observed from Figure 10(b) that, in a tissue (muscle or bone) environment, the PTE first increases and then decreases with frequency, and it reaches a maximum value at $f_{p}=25 \mathrm{MHz}$. For the frequency range of interest, the electromagnetic field loss in the air is almost frequency independent, and as $k$ in the air increases with frequency, the PTE will also increase. However, for the tissue media, the loss of electromagnetic field will increase with frequency, and as a result, the PTE in tissue increases first (owing to the increase of $k$ ) and then decreases (owing to the increase of field loss).

Figure 10(c) shows the SAR-constrained PDL. Owing to the fact that SAR is proportional to the square of the frequency, the PDL within SAR limit thus decreases with frequency monotonically. For the air environment, there is no physical meaning for SAR; therefore, the MPDL in air is not available.

\section{Experimental Verification}

4.1. Measurement Methodology. To verify the simulation results, experiments are carried out, and the detailed view of the Tx and Rx coils is shown in Figure 11. $r_{1}=0.287 \mathrm{~mm}(\# 23$ AWG) and $r_{2}=0.04 \mathrm{~mm}(\# 40 \mathrm{AWG})$ are chosen as the wire radius of the $\mathrm{Tx}$ and $\mathrm{Rx}$ coils, respectively. The experimental setup is similar to that of other works $[17,18]$. The Tx and Rx coils are supported by a nonconducting Plexiglas frame so that the distance between the two coils can be adjusted. The two coils are connected to a vector network analyzer (VNA) via a pair of subminiature version A (SMA) connectors. Since the size of the $\mathrm{Rx}$ coil is close to that of the SMA connector, the magnetic interference caused by the connector will affect the measured results. To overcome this drawback, the deembedding calibration method is adopted [54]. The method subtracted the parasitic components generated by the measuring fixture from the original deviceunder-test (DUT) measurements. The original scattering parameters, $S$, are written as $S_{d u t}$ and can be converted into admittance parameter, $Y$, following the relationship:

$$
Y_{d u t}=\left(G_{0}-S_{d u t}\right)\left(Z_{0} \cdot S_{d u t}+Z_{0}\right)^{-1},
$$

where $G_{0}$ is the unit matrix, $Z_{0}$ is the characteristic impedance matrix of the ports and is expressed as $50 G_{0}$. Similar to $Y_{d u t}, Y_{\text {short }}$ and $Y_{\text {open }}$ can be obtained by using $S_{\text {short }}$ and $S_{\text {open, }}$, where $S_{\text {short }}$ and $S_{\text {open }}$ are the scattering parameters obtained when the circuit is short and open connected, 


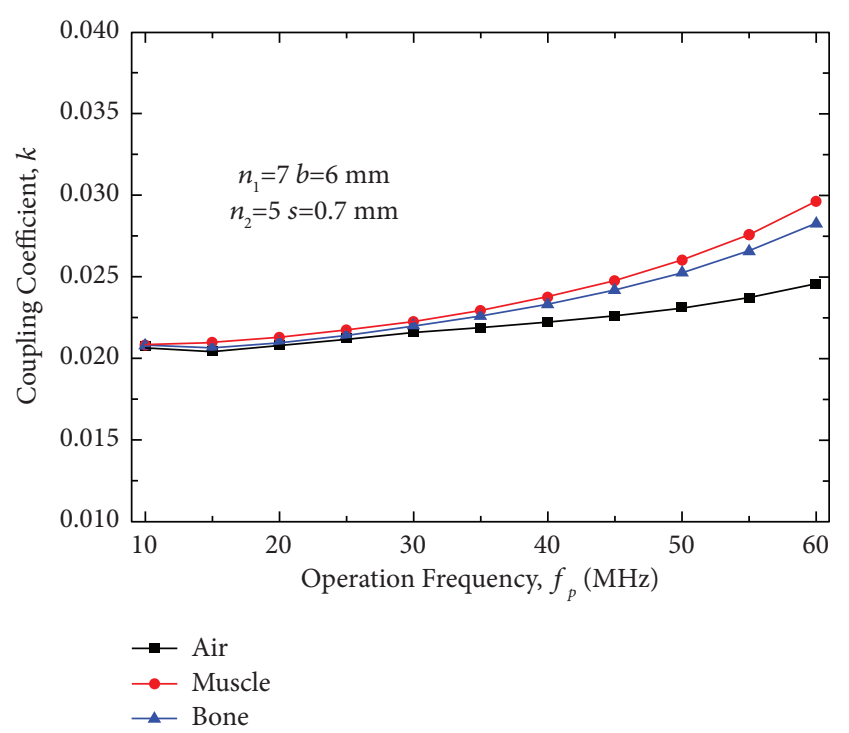

(a)

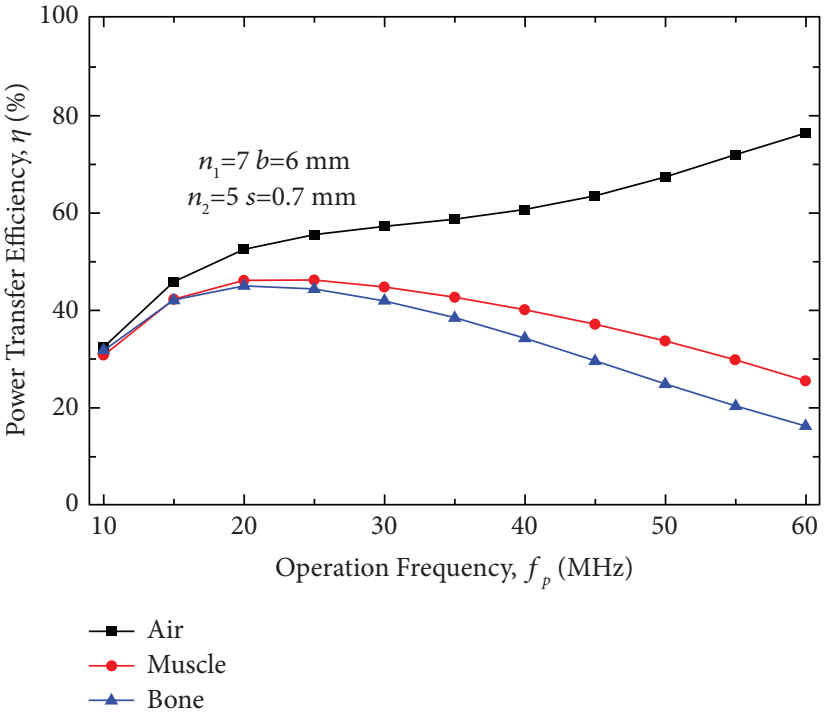

(b)

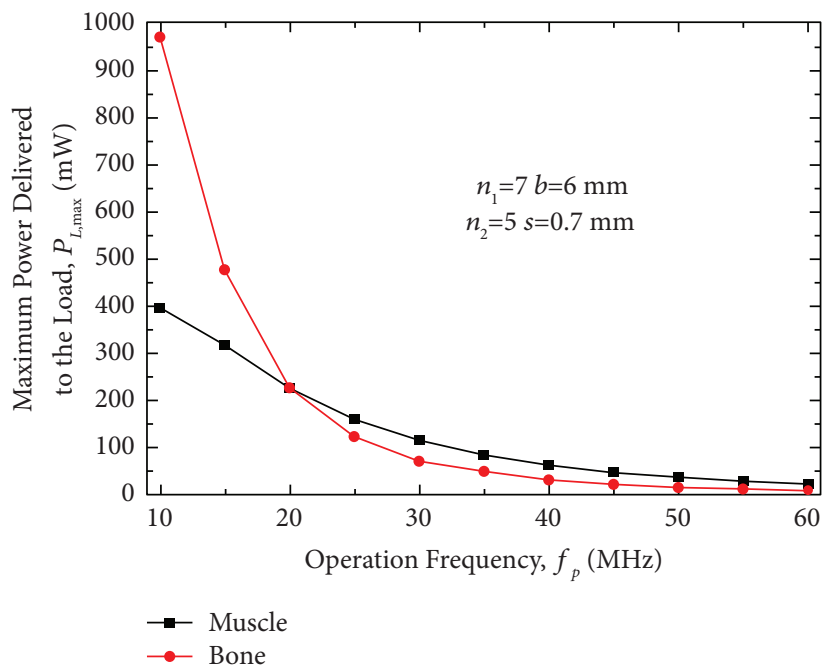

(c)

FIGURE 10: The simulation results of $k$, PTE, and MPDL of a WPT system in different media: (a) the coupling coefficient, (b) the power transfer efficiency, and (c) the SAR-constrained power delivered to the load. $h=20 \mathrm{~mm}, R_{a v}=20 \mathrm{~mm}, R_{2}=5 \mathrm{~mm}, r_{1}=0.287 \mathrm{~mm}$, $r_{2}=0.04 \mathrm{~mm}, n_{1}=7, b=6 \mathrm{~mm}, n_{2}=5$, and $s=0.7 \mathrm{~mm}$.

TABLE 3: Dielectric properties of tissues $\left(f_{p}=25 \mathrm{MHz}\right)$.

\begin{tabular}{lccc} 
Tissue & $\begin{array}{c}\text { Relative } \\
\text { permittivity }\end{array}$ & Conductivity $(\mathrm{S} / \mathrm{m})$ & $\begin{array}{c}\text { Dielectric loss } \\
\text { tangent }\end{array}$ \\
\hline Muscle & 89.3 & 0.56 & 4.53 \\
Bone & 22.5 & 0.05 & 1.62 \\
\hline
\end{tabular}

respectively. After removing the parasitic capacitive coupling and self-capacitance, the $Y$ parameter of the system can be expressed as [55]

$$
Y=\left[\left(Y_{\text {dut }}-Y_{\text {open }}\right)^{-1}-\left(Y_{\text {short }}-Y_{\text {open }}\right)^{-1}\right]^{-1}
$$

The resultant $Y$ parameters are converted into $Z$ parameters, $Z=Y^{-1}$, to obtain [18]

$$
\begin{aligned}
& L_{1}=\frac{\operatorname{Im}\left(Z_{11}\right)}{2 \pi f_{p}}, \\
& L_{2}=\frac{\operatorname{Im}\left(Z_{22}\right)}{2 \pi f_{p}}, \\
& Q_{1}=\frac{\operatorname{Im}\left(Z_{11}\right)}{\operatorname{Re}\left(Z_{11}\right)}, \\
& Q_{2}=\frac{\operatorname{Im}\left(Z_{22}\right)}{\operatorname{Re}\left(Z_{22}\right)}, \\
& Q_{L}=\frac{R_{L}}{\operatorname{Im}\left(Z_{22}\right)},
\end{aligned}
$$




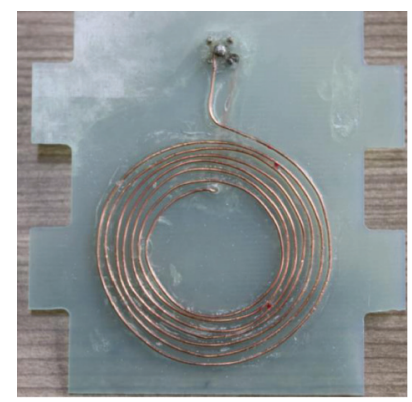

(a)

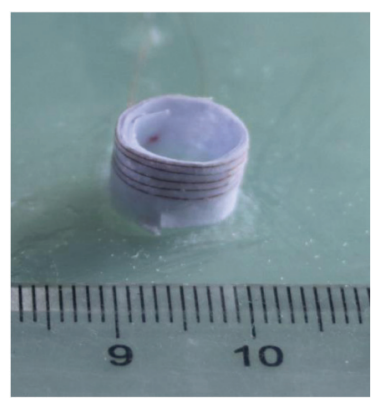

(b)

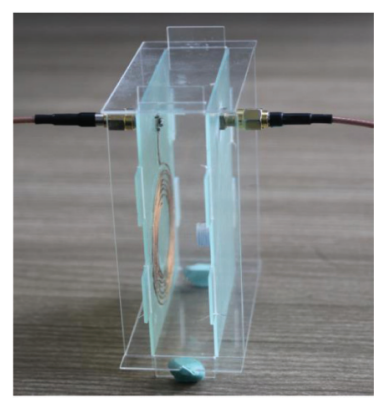

(c)

Figure 11: (a) The detailed view of a planar spiral Tx coil. (b) The cm-sized Rx coil. (c) Inductive link measurement setup with Rx coil in air environments. The Tx and Rx coils are supported by FR4 PCBs and Plexiglas flame and connected to a vector network analyzer by the SMA connectors.

$$
\begin{gathered}
M=\frac{\sqrt{\operatorname{Im}\left(Z_{12}\right) * \operatorname{Im}\left(Z_{21}\right)}}{2 \pi f_{p}}, \\
k=\sqrt{\frac{\operatorname{Im}\left(Z_{12}\right) \cdot \operatorname{Im}\left(Z_{21}\right)}{\operatorname{Im}\left(Z_{11}\right) \cdot \operatorname{Im}\left(Z_{22}\right)}} .
\end{gathered}
$$

4.2. Measured Results of the Proposed Inductive Link WPT System. According to the simulation results, the PTE will reach the maximum value in the muscle around the operation frequency of $25 \mathrm{MHz}$; therefore, $f_{p}=25 \mathrm{MHz}$ is chosen in the experiments. Beef is used to mimic the muscle tissue, and pig's rib bone is used to mimic the bone tissue. The parameters listed in Table 2 are adopted in the experiments.

The $Z$ parameters are obtained by the calculations with $S$ and $Y$ parameters. Having obtained the $Z$ parameters, the self-inductance, the quality factor, and the coupling coefficient of the Tx and Rx coils can be achieved with equations 24-26. The SAR constrained maximum current allowed in the Tx coil, $I_{1, \max }$, can be obtained from Figure 9 . At the frequency of $25 \mathrm{MHz}$, it is $0.384 \mathrm{~A}$ and $0.349 \mathrm{~A}$ for muscle and bone tissues, respectively. With these parameters, the PTE and the SAR-constrained maximum PDL can be calculated with equations (17) and (21). All the results are demonstrated in Table 4.

In Table 4, the measurement shows that the proposed inductive link WPT system can achieve a PTE of $52.35 \%$, $44.14 \%$, and $43.07 \%$ in air, muscle, and bone environments, respectively. The MPDL of the system can reach $145.38 \mathrm{~mW}$ in the muscle tissue and $128.13 \mathrm{~mW}$ in the bone tissue. Agreements between the simulation and measurement are observed.

In order to show the significant contributions of our proposed inductive link WPT system for $\mathrm{cm}$-sized IMDs, we compare our work with others in Table 5. It is easy to observe that the system proposed in this work has advantages in performance.

\section{Discussions}

This work researches the optimizations of coupling coefficient of the inductive link in the WPT system used for IMDs. Simulations and experiments are carried out to test the proposed inductive link. Over $40 \%$ of PTE and $120 \mathrm{~mW}$ MPDL of the WPT system can be obtained in air, muscle, and bone environments, demonstrating the feasibility of the system. However, in real-world applications, there are several issues that need to be addressed.

5.1. Biocompatibility. All implantable devices should be sealed in a biocompatible material to be protected from the harsh environment in the human body. The Rx coil of the WPT system can be embedded in or wrapped around the biomedical implant. For biosafety reasons, if the coil is embedded in an implant, the implant cannot be sealed with metal, as the metal will shield the electromagnetic field from the $\mathrm{Rx}$ coil. In this case, the implant should be encapsulated with a polymeric biocompatible material such as polydimethylsiloxane or silicone. If the $\mathrm{Rx}$ coil is wrapped around an implant, the coil should be covered with the aforementioned biocompatible material.

5.2. Temperature Rise in the Human Body. According to human physiology, some people will go into convulsions at $41^{\circ} \mathrm{C}$ of body temperature, and this value is usually used as the basic temperature restriction [44]. The energy obtained in the receiving loop can be used to recharge the button cell embedded in the implant. With the low power consumption design in IC technology, the power consumed in the $\mathrm{Rx}$ circuit should be kept as low as possible such that the heat generated in the circuit is small, and the recharge process time can be increased.

5.3. The Recharge Cycle of the Button Cell. Generally, the power for a modern cardiac pacemaker is $6 \mu \mathrm{W}$ (according to the reference manual of Nanostim TM, St. Jude Medical) [52]. Assuming that the inductive link WPT system provides a PDL of $120 \mathrm{~mW}$, the recharge efficiency to the button cell is $50 \%$, and the recharge time is 20 minutes; then, the recharged energy can last 3.9 years. Therefore, the button cell is only needed to be recharged once in every 3 years.

5.4. The SAR Safety. In this work, the IMD is intended to be used for left vagus nerve stimulation or cardiac pacemaker, which can be embedded in the chest. Therefore, the local 
TABLe 4: Simulation and measurement results of the inductive link $f_{p}=25 \mathrm{MHz}$.

\begin{tabular}{|c|c|c|c|c|}
\hline Parameters & & Air & Muscle & Bone \\
\hline \multirow{2}{*}{ Tx resistance $\left(R_{T x}, \Omega\right)$} & Simulation & 1.14 & 1.94 & 1.94 \\
\hline & Measurement & 1.23 & 1.87 & 2.10 \\
\hline \multirow{2}{*}{ Tx inductance $\left(L_{1}, \mu \mathrm{H}\right)$} & Simulation & 2.60 & 2.66 & 2.66 \\
\hline & Measurement & 2.57 & 2.54 & 2.55 \\
\hline \multirow{2}{*}{ Tx quality factor $\left(Q_{1}\right)$} & Simulation & 358.12 & 215.28 & 214.97 \\
\hline & Measurement & 327.76 & 213.51 & 190.73 \\
\hline \multirow{2}{*}{ Rx resistance $\left(R_{R x}, \Omega\right)<$} & Simulation & 0.78 & 0.85 & 0.94 \\
\hline & Measurement & 0.86 & 0.88 & 0.91 \\
\hline \multirow{2}{*}{$\mathrm{Rx}$ inductance $\left(L_{2}, \mu \mathrm{H}\right)$} & Simulation & 0.35 & 0.36 & 0.36 \\
\hline & Measurement & 0.34 & 0.33 & 0.34 \\
\hline \multirow{2}{*}{$\mathrm{Rx}$ quality factor $\left(Q_{2}\right)$} & Simulation & 70.97 & 66.48 & 59.98 \\
\hline & Measurement & 61.92 & 59.75 & 58.27 \\
\hline \multirow{2}{*}{ Load quality factor $\left(Q_{L}\right)$} & Simulation & 18.06 & 17.70 & 17.74 \\
\hline & Measurement & 18.78 & 19.02 & 18.86 \\
\hline \multirow{2}{*}{ Coupling coefficient $(k)$} & Simulation & 0.0212 & 0.0217 & 0.0215 \\
\hline & Measurement & 0.0213 & 0.0213 & 0.0221 \\
\hline \multirow{2}{*}{ Power transfer efficiency $(\eta)$} & Simulation & $55.72 \%$ & $46.34 \%$ & $44.40 \%$ \\
\hline & Measurement & $52.35 \%$ & $44.14 \%$ & $43.07 \%$ \\
\hline Peak value of SAR (W/kg) & Simulation & N.A. & 13.58 & 16.42 \\
\hline \multirow{2}{*}{ The SAR-constrained PDL (mW) } & Simulation & N.A. & 160.17 & 123.55 \\
\hline & Measurement & N.A. & 145.38 & 128.13 \\
\hline
\end{tabular}

TABle 5: Performance comparisons with previous works of inductive link WPT system for cm-sized IMDs.

\begin{tabular}{|c|c|c|c|c|c|c|}
\hline \multicolumn{2}{|c|}{ Reference } & [18] & {$[43]$} & [56] & [57] & This work \\
\hline \multirow{3}{*}{$\mathrm{Tx}$} & $R_{\text {out }}(\mathrm{mm})$ & 14 & 12 & 7.5 & 15 & 26 \\
\hline & $R_{\mathrm{in}}(\mathrm{mm})$ & 9 & 4.7 & - & - & 14 \\
\hline & $n_{1}$ & 5 & 2 & 1 & 1 & 7 \\
\hline \multirow{2}{*}{$\mathrm{Rx}$} & $R_{2}(\mathrm{~mm})$ & 0.5 & 5 & 2.9 & 10 & 5 \\
\hline & $n_{2}$ & 7 & 4 & 1 & 1 & 5 \\
\hline \multicolumn{2}{|c|}{$h(\mathrm{~mm})$} & 12 & 10 & 16 & 20 & 20 \\
\hline \multicolumn{2}{|c|}{$\begin{array}{l}\text { Frequency } \\
(\mathrm{MHz})\end{array}$} & 20 & 13.56 & 300 & 200 & 25 \\
\hline \multicolumn{2}{|c|}{$R_{L}(\mathrm{k} \Omega)$} & 0.25 & 0.5 & 10 & 0.47 & 1 \\
\hline \multicolumn{2}{|c|}{ PTE, $\eta(\%)$} & 1.4 & 27.7 & 2.8 & 47.6 & 44.1 \\
\hline \multicolumn{2}{|c|}{ MPDL (mW) } & 2.2 & - & 0.8 & 29.8 & 145.4 \\
\hline
\end{tabular}

SAR value of $2 \mathrm{~W} / \mathrm{kg}$ is adopted. The Tx coil is set to be $2 \mathrm{~mm}$ away from the skin. The Tx coil can be embedded in wearable things, such as cloth, and the 2-mm separation space should be guaranteed. In practice, the local value of SAR should be measured with equipment such that the security is absolutely assured.

In the practical applications, the aforementioned issues should be carefully explored. Furthermore, the magnetic field distribution results for all verification cases on $\mathrm{Rx}$ movement and the changes in tissue characteristics are of interests. Future researches may be focused on all of these issues.

\section{Conclusions}

The analytical expression for the coupling coefficient of the inductive link is presented, and the optimizations of the coupling coefficient are provided. Based on the optimization results, an inductive link for the WPT system is proposed, and simulation and measurement results are obtained. Measurement demonstrates that, in a muscle environment, the system can achieve a PTE of $44.14 \%$, with the SAR-constrained PDL of $145.38 \mathrm{~mW}$.

With the rapid progress in IC technologies, the IMDs can be miniaturized to $\mathrm{mm}$-scale and can be implanted for deep brain stimulation [5]. The advancement in biocompatible and novel nanomaterial electrode interfaces has made it possible to attach the IMDs directly to the neural tissues [42]. However, for some specific applications, such as a cardiac pacemaker or nerve stimulator, IMDs in the $\mathrm{cm}$ scale are preferred. With the advancement in modern techniques, this target will surely be implemented in the near future. For both convenience and comfort, these IMDs are usually powered by a button cell, as such, the scale of the IMD is restricted by the size of the cell. The inductive link WPT system proposed in this work is to recharge the cell; with this technology, surgery of replacing the IMD is avoided and the patients can be benefited both physiologically and economically.

\section{Data Availability}

The data are available from the corresponding author upon request (gxhu@fudan.edu.cn).

\section{Conflicts of Interest}

The authors declare that they have no conflicts of interest.

\section{Acknowledgments}

This work was supported in part by the Chinese Postdoctoral Science Foundation (Grant no. 2020M681168), in part by the National Natural Science Foundation of China (Grant no. 62101135), and in part by the Shanghai Pujiang Program (Grant no. 20PJ1400900). 


\section{References}

[1] G. J. Suaning and N. H. Lovell, "CMOS neurostimulation ASIC with 100 channels, scaleable output, and bidirectional radio-frequency telemetry," IEEE Transactions on Biomedical Engineering, vol. 48, no. 2, pp. 248-260, 2001.

[2] M. Schwarz, R. Hauschild, B. J. Hosticka et al., "Single-chip CMOS image sensors for a retina implant system," IEEE Transactions on Circuits and Systems II: Analog and Digital Signal Processing, vol. 46, no. 7, pp. 870-877, 1999.

[3] G. A. Kendir, W. T. Liu, G. X. Wang et al., "An optimal design methodology for inductive power link with class-E amplifier," IEEE Transactions on Circuits and Systems I: Regular Papers, vol. 52, no. 5, pp. 857-866, 2005.

[4] S. A. Mirbozorgi, P. Yeon, and M. Ghovanloo, "Robust wireless power transmission to $\mathrm{mm}$-sized free-floating distributed implants," IEEE Transactions on Biomedical Circuits and Systems, vol. 11, no. 3, pp. 1-11, 2017.

[5] Y. Jia, S. A. Mirbozorgi, B. Lee et al., "A mm-sized free-floating wirelessly powered implantable optical stimulation device," IEEE Transactions on Biomedical Circuits and Systems, vol. 13, no. 4, pp. 608-618, 2019.

[6] P. Yeon, S. A. Mirbozorgi, J. Lim, and M. Ghovanloo, "Feasibility study on active back telemetry and power transmission through an inductive link for millimeter-sized biomedical implants," IEEE Transactions on Biomedical Circuits and Systems, vol. 11, no. 6, pp. 1366-1376, 2017.

[7] Y. Cheng, G. Wang, and M. Ghovanloo, "Analytical modeling and optimization of small solenoid coils for millimeter-sized biomedical implants," IEEE Transactions on Microwave Theory and Techniques, vol. 65, no. 3, pp. 1024-1035, 2017.

[8] H. Bhamra, J. W. Tsai, and Y. W. Huang, "A sub-cubic millimeter wireless implantable intraocular pressure monitor microsystem," IEEE Transactions on Biomedical Circuits and Systems, vol. 11, no. 6, pp. 1-12, 2017.

[9] K. Chul and H. Sohmyung, "A 144-MHz fully integrated resonant regulating rectifier with hybrid pulse modulation for mm-sized implants," IEEE Journal of Solid-State Circuits, vol. 52, no. 11, pp. 3043-3055, 2017.

[10] A. Ibrahim, M. Meng, and M. Kiani, "A comprehensive comparative study on inductive and ultrasonic wireless power transmission to biomedical implants," IEEE Sensors Journal, vol. 18, no. 9, pp. 3813-3826, 2018.

[11] M. Meng and M. Kiani, "Design and optimization of ultrasonic wireless power transmission links for millimeter-sized biomedical implants," IEEE Transactions on Biomedical Circuits and Systems, vol. 11, no. 1, pp. 98-107, 2017.

[12] T. C. Chang, M. J. Weber, J. Charthad, S. Baltsavias, and A. Arbabian, "End-to-end design of efficient ultrasonic power links for scaling towards submillimeter implantable receivers," IEEE Transactions on Biomedical Circuits and Systems, vol. 12, no. 5, pp. 1100-1111, 2018.

[13] J. Lim, T. S. Khwaja, and J. Ha, "Wireless optical power transfer system by spatial wavelength division and distributed laser cavity resonance," Optics Express, vol. 27, no. 12, pp. A924-A935, 2019.

[14] Y.-S. Lin and K.-S. Lan, "RF-DC converter and DC-DC converter for UHF and microwave wireless power transfer (UWPT/MWPT)," Analog Integrated Circuits and Signal Processing, vol. 101, no. 3, pp. 585-599, 2019.

[15] S. Hu, J. Bao, G. Hu, D. Ta, R. Liu, and L. Zheng, "Electric field and transmitting power analysis of segmented and unsegmented loop antennas for transcutaneous power transfer," IEEE Transactions on Antennas and Propagation, vol. 69, no. 6, pp. 3485-3492, 2021.
[16] H. Zhang, F. Lu, H. Hofmann, W. Liu, and C. C. Mi, "Six-plate capacitive coupler to reduce electric field emission in large airgap capacitive power transfer," IEEE Transactions on Power Electronics, vol. 33, no. 1, pp. 665-675, 2018.

[17] D. Ahn and M. Ghovanloo, "Optimal design of wireless power transmission links for millimeter-sized biomedical implants," IEEE Transactions on Biomedical Circuits and Systems, vol. 10, no. 1, pp. 125-137, 2016.

[18] A. Ibrahim and M. Kiani, "A figure-of-merit for design and optimization of inductive power transmission links for millimeter-sized biomedical implants," IEEE Transactions on Biomedical Circuits and Systems, vol. 10, no. 6, pp. 1100-1111, 2016.

[19] S. I. Babic and C. Akyel, "An improvement in the calculation of the self-inductance of thin disk coils with air-core," WSEAS Transactions on Circuits and Systems, vol. 3, no. 8, pp. 1621-1626, 2004.

[20] S. I. Babic and C. Akyel, "Magnetic force between circular filament coil and massive circular coil with rectangular cross section," WSEAS Transactions on Circuits and Systems, vol. 4, no. 6, pp. 610-617, 2005.

[21] G. Zhong and C. Kok Koh, "Exact closed form formula for partial mutual inductance of on-chip interconnects," in Proceedings of the ICCD, pp. 1-6, Freiburg, Germany, September 2002.

[22] J. T. Conway, "Exact solutions for the magnetic fields of axisymmetric solenoids and current distributions," IEEE Transactions on Magnetics, vol. 37, no. 4, pp. 2977-2988, 2001.

[23] S. Babic, C. Akyel, and S. J. Salon, "New procedures for calculating the mutual inductance of the system: filamentary circular coil-massive circular solenoid," IEEE Transactions on Magnetics, vol. 38, no. 5, pp. 1131-1134, 2003.

[24] S. Babic, S. Salon, and C. Akyel, "The mutual inductance of two thin coaxial disk coils in air," IEEE Transactions on Magnetics, vol. 40, no. 2, pp. 822-825, 2004.

[25] S. Babic and C. Akyel, "An improvement in calculation of the self-and mutual inductance of thin-wall solenoids and disk coils," IEEE Transactions on Magnetics, vol. 36, no. 4, pp. 678-684, 2000.

[26] D. Yu and K. S. Han, "Self-inductance of air-core circular coils with rectangular cross-section," IEEE Transactions on Magnetics, vol. 23, no. 6, pp. 3916-3921, 1987.

[27] A. V. Kildishev, "Application of spheroidal functions in magnetostatics," IEEE Transactions on Magnetics, vol. 40, no. 2, pp. 846-849, 2004.

[28] C. A. Borghi, U. Reggiani, and G. Zama, "Calculation of mutual inductances by means of the toroidal multipole expansion method," IEEE Transactions on Magnetics, vol. 25, no. 4, pp. 2992-2994, 1989.

[29] M. W. Garrett and M. Wayne, "Calculation of fields, forces, and mutual inductances of current systems by elliptic integrals," Journal of Applied Physics, vol. 34, no. 9, pp. 2567-2573, 1963.

[30] Y. Cheng and Y. Shu, "A new analytical calculation of the mutual inductance of the coaxial spiral rectangular coils," IEEE Transactions on Magnetics, vol. 50, no. 4, pp. 1-6, 2014.

[31] K. B. Ki-Bong Kim, E. Levi, Z. Zabar, and L. Birenbaum, "Mutual inductance of noncoaxial circular coils with constant current density," IEEE Transactions on Magnetics, vol. 33, no. 5, pp. 4303-4309, 1997.

[32] C. Akyel, S. Babic, and S. Kincic, "New and fast procedures for calculating the mutual inductance of coaxial circular coils (disk coil-circular coil)," IEEE Transactions on Magnetics, vol. 38, no. 5, pp. 1367-1369, 2002. 
[33] S. I. Babic and C. Akyel, "Calculating mutual inductance between circular coils with inclined axes in air," IEEE Transactions on Magnetics, vol. 44, no. 7, pp. 1743-1750, 2008.

[34] H. Greenhouse, "Design of planar rectangular microelectronic inductors," IEEE Transactions on Parts, Hybrids, and Packaging, vol. 10, no. 2, pp. 101-109, 1974.

[35] A. E. Ruehli, "Inductance calculations in a complex integrated circuit environment," IBM Journal of Research and Development, vol. 16, no. 5, pp. 470-481, 1972.

[36] S. S. Mohan, M. del Mar Hershenson, S. P. Boyd, and T. H. Lee, "Simple accurate expressions for planar spiral inductances," IEEE Journal of Solid-State Circuits, vol. 34, no. 10, pp. 1419-1424, 1999.

[37] S. Butterworth, "On the self-inductance of single-layer flat coils," Proceedings of the Physical Society of London, vol. 32, no. 1, pp. 31-37, 1919.

[38] U.-M. Jow and M. Ghovanloo, "Design and optimization of printed spiral coils for efficient transcutaneous inductive power transmission," IEEE Transactions on Biomedical Circuits and Systems, vol. 1, no. 3, pp. 193-202, 2007.

[39] C. Xiao, D. Cheng, and K. Wei, "An LCC-C compensated wireless charging system for implantable cardiac pacemakers: theory, experiment, and safety evaluation," IEEE Transactions on Power Electronics, vol. 33, no. 6, pp. 4894-4905, 2018.

[40] C. Liu, C. Jiang, J. Song, and K. T. Chau, "An effective sandwiched wireless power transfer system for charging implantable cardiac pacemaker," IEEE Transactions on Industrial Electronics, vol. 66, no. 5, pp. 4108-4117, 2019.

[41] A. Kurs, A. Karalis, R. Moffatt, J. D. Joannopoulos, P. Fisher, and M. Soljacic, "Wireless power transfer via strongly coupled magnetic resonances," Science (New York, N.Y.), vol. 317, no. 5834, pp. 83-86, 2007.

[42] K. Agarwal, R. Jegadeesan, Y. X. Guo, and N. V. Thakor, "Wireless power transfer strategies for implantable bioelectronics," IEEE reviews in biomedical engineering, vol. 10, pp. 136-161, 2017.

[43] U.-M. Jow and M. Ghovanloo, "Modeling and optimization of printed spiral coils in air, saline, and muscle tissue environments," IEEE Transactions on Biomedical Circuits and Systems, vol. 3, no. 5, pp. 339-347, 2009.

[44] F. W. Grover, "Methods of calculation inductances," in Inductance Calculations, pp. 6-17, D. Van Nostrand, Dover, NY, USA, 1946.

[45] A. Danisi, A. Masi, R. Losito, and Y. Perriard, "Electromagnetic analysis and validation of an ironless inductive position sensor," IEEE Transactions on Instrumentation and Measurement, vol. 62, no. 5, pp. 1267-1275, 2013.

[46] A. Danisi, A. Masi, R. Losito, and Y. Perriard, "Electromagnetic model of an ironless inductive position sensor," in Proceedings of the IEEE I2MTC, pp. 69-74, Graz, Austria, May 2012.

[47] R. Dengler, "Self inductance of a wire loop as a curve integral," Advanced Electromagnetics, vol. 5, no. 1, pp. 1-8, 2016.

[48] R. Shadid and S. Noghanian, "A literature survey on wireless power transfer for biomedical devices," International Journal of Antennas and Propagation, vol. 2018, Article ID 4382841, 11 pages, 2018.

[49] "IEEE recommended practice for measurements and computations of radio frequency electromagnetic fields with respect to human exposure to such fields, $100 \mathrm{kHz}-300 \mathrm{GHz}$, IEEE Std C95.3-2002, ," 2002.

[50] A. Christ, M. Douglas, J. Nadakuduti, and N. Kuster, "Assessing human exposure to electromagnetic fields from wireless power transmission systems," Proceedings of the IEEE, vol. 101, no. 6, pp. 1482-1493, 2013.
[51] C. Rong, X. He, Y. Wu et al., "Optimization design of resonance coils with high misalignment tolerance for drone wireless charging based on genetic algorithm," IEEE Transactions on Industry Applications, p. 1, 2021.

[52] A. Zurbuchen, A. Haeberlin, A. Pfenniger et al., "Towards batteryless cardiac implantable electronic devices-the Swiss way," IEEE Transactions on Biomedical Circuits and Systems, vol. 11, no. 1, pp. 78-86, 2017.

[53] S. Gabriel, R. W. Lau, and C. Gabriel, "The dielectric properties of biological tissues: III. Parametric models for the dielectric spectrum of tissues," Physics in Medicine and Biology, vol. 41, no. 11, pp. 2271-2293, 1996.

[54] H. Cho and D. E. Burk, "A three-step method for the deembedding of high-frequency S-parameter measurements," IEEE Transactions on Electron Devices, vol. 38, no. 6, pp. 1371-1375, 1991.

[55] M. Koolen, J. Geelen, and M. Versleigen, "An improved de-embedding technique for on-wafer high-frequency characterization," in Proceedings of the Bipolar Circuits and Technology Meeting, pp. 188-191, Minneapolis, MN, USA, September 1991.

[56] R. Muller, H.-P. Le, W. Li et al., "A minimally invasive 64-channel wireless $\mu \mathrm{ECoG}$ implant," IEEE Journal of Solid-State Circuits, vol. 50, no. 1, pp. 344-359, 2015.

[57] D. Ahn and S. Hong, "Wireless power transmission with self-regulated output voltage for biomedical implant," IEEE Transactions on Industrial Electronics, vol. 61, no. 5, pp. 2225-2235, 2014. 